\title{
【記念請演】
}

\section{Mutant Mice as a Tool for Investigation of Mast Cell Differentiation*}

\author{
Yukihiko KITAMURA \\ Department of Pathology, Osaka University Medical School, Osaka
}

\begin{abstract}
Summary
Mutant mice are an invaluable tool for investigation of mast cell differentiation. Giant granules of $b g^{J} / b g^{J}$ mice are used as a marker to identify the origin of mast cells. Mice of $W / W^{v}$, $S l / S l^{d}$ and $m i / m i$ genotypes lack mast cells, and are useful not only for studying the differentiation processes but also clarifying the regulation mechanisms. Although mast cells had been considered to be derived from undifferentiated mesenchymal cells, we showed that mast cells are a unique class of blood cells. Unlike most blood cells, undifferentiated precursors of mast cells migrate in the bloodstream, invade tissues, proliferate there and then differentiate. Even after differentiation, some mast cells may proliferate. Moreover, after functioning (i.e., degranulation), some mast cells may proliferate and produce granules again. Differentiation of mast cells is promoted by both $\mathrm{T}$ cells and fibroblasts and is suppressed by differentiated mast cells. Mast cell deficiency of $W / W^{v}, S l / S l^{d}$ and $m i / m i$ mice is attributed to defects of fibroblast-dependent mechanisms, and deficient mast-cell reaction in nude athymic mice to depletion of $\mathbf{T}$ cells.
\end{abstract}

\section{Introduction}

Mast cells have immunoglobulin E (IgE) receptors on their surface, and their immunological activity is mediated through these IgE receptors. Binding of antigens to IgE molecules results in formation of linkages between $\operatorname{IgE}$ receptors, and then release of the granules themselves or chemical mediators in the granules (proteoglycans, proteases, histamine, etc.). This process constitutes an important step in the immediate hypersensitivity reaction, which occurs in allergic diseases such as bronchial asthma, and allergic rhinitis. In addition to having a role in allergic diseases, mast cells have a physiological role as effectors of host defense mechanisms in intestinal helminth infection and dermal tick infestation. ${ }^{1-3)}$

\footnotetext{
*Presented as a memorial lecture for receiving the Osaka Science Award at the 6th General Meeting of the Japanese Association of Animal Models for Human Diseases.

Address reprint requests to Yukihiko Kitamura, M.D., Department of Pathology, Osaka University Medical School, Nakanoshima 4-3-57, Kita-ku, Osaka, 530 Japan
}

\section{Mutant animals}

Mice of the $b g^{J} / b g^{J}$ genotype is useful for studies on mast-cell differentiation, since the giant granules of this mutant can be used as a marker in investigating the origin of mast cells. Intravenous injection of $b g^{J} / b g^{J}$ bone marrow cells into lethally irradiated congenic mice resulted in development of mast cells with giant granules, indicating the bone marrow origin of mast cells.4) We recently found a rat mutant comparable to $b g^{J} / b g^{J}$ mice. ${ }^{5)}$ This mutant rat may be useful for investigating differentiation processes of mast cells in rats. We found that mutant mice of either $W / W^{v}$ or $S l / S l^{d}$ genotype have no mast cells. $\left.{ }^{6}, 7\right)$ The absence is attributed to a defect of precursor cells in $W / W^{v}$ mice, ${ }^{6}$ ) and to a defect of the microenvironment necessary for differentiation of mast cells in $S l / S l^{d}$ mice. ${ }^{7)}$

We analysed the mechanisms of mast cell deficiency of $W / W^{v}$ and $S l / S l^{d}$ mice by co-culturing mast cells and fibroblasts. $T$ cells of both $W / W^{v}$ and $S l / S l^{d}$ mice can produce interleukin (IL)-3 and IL-4. ${ }^{1,8)}$ Mast cells develop when bone marrow cells of either $W / W^{0}$ or 
$S l / S l^{d}$ mice are cultured in the medium containing IL-3. Therefore, these $T$ cell factors do not appear to be involved in the actions of the $W$ and $S l$ mutant genes. Fibroblast cell lines established from normal $(+/+)$ mouse embryos supported the growth of cultured mast cells derived from $+1+$ mice. In contrast, these fibroblast cell lines did not support cultured mast cells derived from $W / W^{v}$ mice, suggesting that the $W$ gene product expressed on the surface of mast cells is mandatory for the fibroblast-dependent proliferation. ${ }^{1,8,9)}$

Cultured mast cells derived from $S / / S^{d}$ mice were maintained as well by $+/+$ embryoderived fibroblast cell lines as were cultured mast cells derived from $+/+$ mice, indicating the normal response of $S l / S l^{d}$ mast cells to fibroblasts. On the other hand, the fibroblast cell lines derived from $S l / S l^{d}$ embryos did not induce the proliferation of $+/+$ mast cells, suggesting that $S l$ gene product expressed by fibroblasts is indispensable for the fibroblastdependent proliferation of cultured mast cells. ${ }^{9}{ }^{90}$ ) Since the $W$ locus was shown to be identical with the c-kit proto-oncogene that encodes a tyrosine kinase receptor, ${ }^{11,12)}$ the product of $S l$ locus may be the ligand for the receptor encoded by the $W$ locus.

3T3 fibroblast cell lines derived from $+/+$ embryos did not affect the IL-3-dependent proliferation of cultured mast cells, but $S \mathrm{Sl} S \mathrm{I}^{d}$ embryo-derived 3T3 fibroblast cell lines significantly suppressed the proliferation. Close cellto-cell contact was necessary for the suppression. The NWS1 fibroblast cell line was established from the spleen cells of adult $+1+$ mouse. Although the NWS1 cell line had no supporting effect on the proliferation of mast cells in the absence of diffusible growth factors, it did not suppress the proliferation of mast cells induced by IL-3. The present result suggests that a product of mutant $S l$ genes may be involved in the suppressive activity of $S l / S l^{d}$ mouse embryoderived 3T3 cells. ${ }^{10}$

Mice of $m i / m i$ genotype are osteopetrotic and are deficient in mast cells. As described in the case of $W / W^{v}$ and $S l / S l^{d}$ mice, mast cells did develop when spleen cells of mi/mi mice were cultured in the presence of IL-3. However, $m i / m i$ mouse-derived cultured mast cells did not proliferate when they were co-cultured with $+/+$ fibroblasts. Since fibroblasts derived from the skin of $m i / m i$ mice supported the proliferation of $+1+$ mast cells, the mast cell deficiency of $m i / m i$ mice was attributed to the inability of $m i / m i$ mast cells to respond to the proliferative stimulus presented by $+/+$ fibroblasts. ${ }^{13)}$

Although nude athymic $(n u / n u)$ mice possess at least normal number of mast cells, they are useful for understanding the role of $\mathrm{T}$ cells for mast cell development. $T$ cells produce IL-3 and IL-4, and these growth factors support development of mast cells in vitro. ${ }^{14,15)}$ Moreover, IL-3 induces mast-cell reaction in the intestine of mice. ${ }^{1,15}$ In fact, a prominent increase of mast cells occurs at the infection site of Trichinella spriralis in normal control mice but not in $n u / n u$ mice. ${ }^{2)}$ The lack of mast-cell reaction after parasite infection was normalized by the previous grafting of thymus to the $n u / n u$ mice.

\section{Differentiation processes}

Although mast cells were originally considered to be derived from undifferentiated mesenchymal cells, we demonstrated that mast cells are the progeny of multipotential stem cells. ${ }^{16)}$ Most of the progenies of multipotential stem cells leave hematopoietic tissue after they have maturated. However, mast cells do not complete their differentiation in hematopoietic tissue although they are progeny of stem cells. The mast-cell precursors invade the connective or mucosal tissues, proliferate and differentiate into morphologically identifiable mast cells. ${ }^{17}$,18)

Most progenies of multipotential stem cells lose their proliferative potential when they become differentiated morphologically. In contrast, mast cells that differentiate in the connective tissue have a long life span (probably more than one year), and at least a portion of morphologically identifiable mast cells have an appreciable proliferative potential. ${ }^{19)}$ Moreover, mast cells can proliferate and reproduce granules after degranulation (Fig. 1). ${ }^{20}$ )

The differentiation process of mast cells has some similarities with those of macrophages and $T$ cells. Precursors of macrophages migrate in the bloodstream as monocytes, and proliferate after their arrival in the connective tissue. Both 


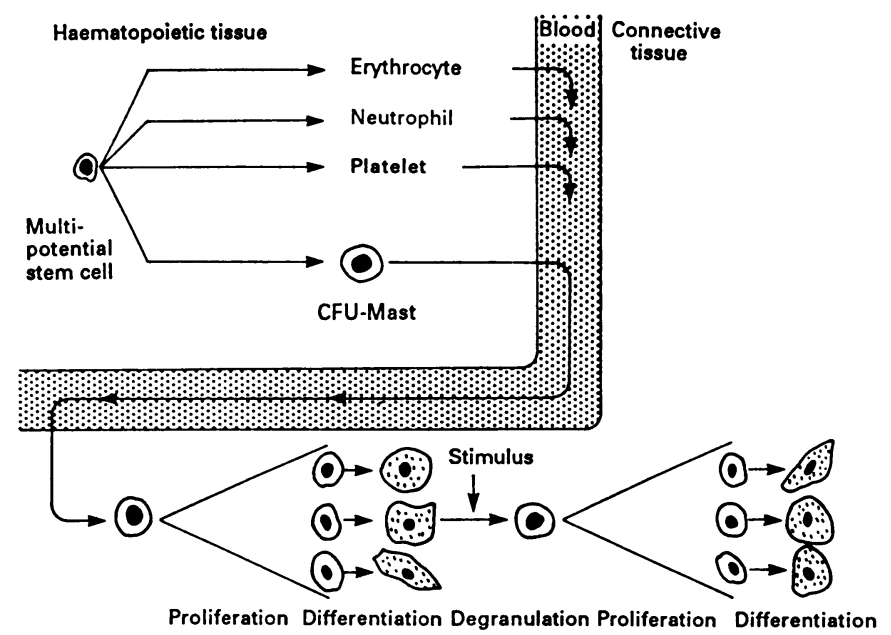

Fig. 1. A scheme showing differentiation processes of mast cells in the connective tissue of mice.

mast cells and $\mathbf{T}$ cells can proliferate after maturation.

\section{Inhibition of differentiation}

Unpleasant symptoms accompany the overproduction of mast cells such as are observed in patients with urticaria pigmentosa, a benign skin tumor of mast cells. This fact suggests the possibility that suppression as well as induction of mast cell differentiation may be an important normal regulatory mechanism. Peritoneal mast cells of mice were eradicated by intraperitoneal injection of distilled water, and the regeneration process was analysed by estimating the changes in numbers of mast-cell precursors and morphologically identifiable mast cells. The presence of morphologically identifiable mast cells appears to suppress the invasion of the precursors from the blocd to the peritoneal cavity and to inhibit the differentiation of the precursors into morphologically identifiable mast cells. ${ }^{21}$ )

Since the injection of large volume of distilled water did not appear to be physiological, another experimental system was developed to confirm the inhibitory effect of mast cells on invasion and differentiation of precursor cells; $W / W^{v}$ mice were used as recipients. ${ }^{22}$ When bone marrow cells of $+/+$ mice were intravenously injected, the concentration of mast-cell precursors increased in the peritoneal cavity of
$W / W^{v}$ mice and became several times greater than that of nontreated $+1+$ mice. This increase was suppressed by a prior intraperitoneal injection of $b g^{J} / b g^{J}$-type cultured mast cells, which were used to identify the $+/+$ origin of mast-cell precursors. The differentiation of $+1+$-type precursor cells to morphologically identifiable mast cells was also suppressed by the intraperitoneal injection of $b g^{J} / b g^{J}$-type cultured mast cells. ${ }^{22)}$

The diffusion chamber technique was used to investigate the mechanism of the suppressive effect. Cultured mast cells derived from $+1+$ mice and NIH/3T3 fibroblasts were coinoculated to diffusion chambers, and the diffusion chambers were implanted into the peritoneal cavity of either $+/+$ or $W / W^{v}$ mice. The magnitude of proliferation of mast cells in diffusion chambers was not significantly influenced by the genotype of chamber recipients (i.e., the presence or absence of mast cells in the peritoneal cavity), suggesting that the suppressive effect may be mediated by direct contact between mast cells. ${ }^{23)}$

\section{Phenotypic change between subpopulations}

Mast cells may be classified into at least two phenotypically distinct populations; connective tissue-type mast cells (CTMC) and mucosal mast cells (MMC). ${ }^{1)}$ CTMC are present in the skin, peritoneal cavity, and the 
muscularis propria of the stomach, among other sites. CTMC contain heparin proteoglycan and store relatively large amounts of histamine. In contrast, $\mathrm{MMC}$ are prominent in the mucosal layer of the gastrointestinal tract, and contain chondroitin sulfate proteoglycan and relatively small quantities of histamine. Recently, we estimated the turnover of CTMC and MMC by determining proportion of mast cells that were labeled with continuously administered bromodeoxyuridine. Bromodeoxyuridine was added to drinking water, and its incorporation was determined immunohistochemically. The turnover of MMC in the gastric mucosa was significantly faster than that of CTMC. ${ }^{24)}$

We investigated the interrelation of CTMC and $\mathrm{MMC}$ by combined in vitro and in vivo approaches. The phenotypic expression of bone marrow-derived cultured mast cells was similar to that of MMC. But when cultured mast cells were injected into the peritoneal cavity of $W / W^{v}$ mice, mast cells with the histochemical and electron microscopical features of CTMC appeared. ${ }^{25},{ }^{26)}$ Cultured mast cells synthesized chondroitin sulfate proteoglycan, but mast cells recovered from the peritoneal cavity of the $W / W^{v}$ mice synthesized heparin proteoglycan. ${ }^{27)}$ The content of histamine increased to a level comparable to that of CTMC. ${ }^{25}$ ) This suggests that MMC may change to CTMC in an appropriate tissue environment.

The phenotypic change occurs in the opposite direction as well. $\left.{ }^{28},{ }^{29}\right)$ When purified peritoneal CTMC of $+/+$ mice were cultured in methylcellulose with IL-3 and IL-4, about $25 \%$ of the CTMC formed colonies. When mast cells from each colony were grown in suspension culture, they sythnesized chondroitin sulphate proteoglycan. Then these MMC-like cultured mast cells derived from a peritoneal CTMC were injected into the peritoneal cavity of $W / W^{v}$ mice; the adoptively transferred mast-cell population again acquired the CTMC-like phenotype. Thus, clonal mast-cell populations, initially derived from a single peritoneal CTMC, exhibited multiple and bidirectional alterations between CTMC-like and MMC-like phenotypes. ${ }^{29)}$

Acknowledgement: I would like to express my gratitude to all the members in the Department of Pathology. The studies described in the present review were supported by the grants from the Ministry of Education, Science and Culture, the Ministry of Health and Welfare, the Hoansha Foundation, the Cell Science Research Foundation, and the Osaka Science Award.

\section{REFERENCES}

1) Kitamura, Y.: Annu. Rev. Immunol., 7, 59 (1989)

2) Matsuda, H., Watanabe, N., Kiso, Y., Hirota, S., Ushio, H., Kanan, Y., Azuma, M., Koyama, H. and Kitamura, Y.: J. Immunol., 144, 259 (1990)

3) Ruitenberg, E.J. and Elgersma, A.: Nature, 264, 258 (1976)

4) Kitamura, Y., Shimada, M., Hatanaka, K. and Miyano, Y.: Nature, 268, 442 (1977)

5) Nishimura, M., Inoue, M., Nakano, T., Nishikawa, T., Miyamoto, M., Kobayashi, T. and Kitamura, Y.: Blood, 74, 270 (1989)

6) Kitamura, Y., Go, S. and Hatanaka, K.: Blood, 52, 447 (1978)

7) Kitamura, Y. and Go, S.: Blood, 53, 492 (1979)

8) Fujita, J., Nakayama, H., Onoue, H., Ebi, Y., Kanakura, Y., Kuriu, A. and Kitamura, Y.: Blood, 72, 463 (1988)

9) Kitamura, Y., Nakayama, H. and Fujita, J.: Mast Cell and Basophil Differentiation and Function in Health and Disease (Galli, S.J. and Austen, K.F., eds.), Raven: Press, New York, pp. 15-25 (1989)

10) Onoue, H., Ebi, Y., Nakayama, H., Ru, X.M., Kitamura, Y. and Fujita, J.: Blood, 74, 1557 (1989)

11) Chabot, B., Stephenson, D.A., Chapman, V.M., Besmer, P. and Bernstein, A.: Nature, 335, 88 (1988)

12) Geissler, E.N., Malanie, A.R. and Housman, D.E.: Cell, 55, 185 (1988)

13) Ebi, Y., Kasugai, T., Seino, Y., Onoue, H., Kanemoto, T. and Kitamura, Y.: Blood, 75, 1247 (1990)

14) Hamaguchi, Y., Kanakura, Y., Fujita, J., Takeda, S., Nakano, T., Tarui, S., Honjo, T. and Kitamura, Y.: J. Exp. Med., 165, 268 (1987)

15) Kitamura, Y. and Matsuda, H.: Cytokines, 2, 73 (1989)

16) Kitamura, Y., Yokoyama, M., Matsuda, H., Ohno, T. and Mori, K.J.: Nature, 291, 159 (1981) 
17) Kitamura, Y., Matsuda, H. and Hatanaka, K.: Nature, 281, 154 (1979)

18) Kitamura, Y. and Fujita, J.: Bioessays, 10, 193 (1989)

19) Sonoda, T., Kanayama, Y., Hara, H., Hayashi, C., Tadokoro, M., Yonezawa, T. and Kitamura, Y.: J. Exp. Med., 160, 138 (1984)

20) Kuriu, A., Sonoda, S., Kanakura, Y., Jozaki, K., Yamatodani, A. and Kitamura, Y.: Blood, 74, 925 (1989)

21) Kanakura, Y., Kuriu, A., Waki, N., Nakano, T., Asai, H., Yonezawa, T. and Kitamura, Y.: Blood, 71, 573 (1988)

22) Waki, N., Kitamura, Y., Kanakura, Y., Asai, H. and Nakano, T.: Exp. Hematol., 18, 138 (1990)

23) Ru, X.M., Onoue, H., Nakayama, H., Ebi, Y., Fujita, J., Kasugai, T. and Kitamura, Y.: Exp. Hematol., 18, 238 (1990)

24) Fukuzumi, T., Waki, N., Kanakura, Y.,
Nagoshi, J., Hirota, S., Yoshikawa, K. and Kitamura, Y.: Exp. Hematol., in press.

25) Nakano, T., Sonoda, T., Hayashi, C., Yamatodani, A., Kanayama, Y., Asai, H., Yonezawa, T., Kitamura, Y. and Galli, S.J.: $J$. Exp. Med., 162, 1025 (1985)

26) Nakano, T., Kanakura, Y., Asai, H. and Kitamura, Y.: J. Immunol., 138, 544 (1987)

27) Otsu, K., Nakano, T., Kanakura, Y., Asai, H., Katz, H.R., Austen, K.F., Stevens, R.L., Galli, S.J. and Kitamura, Y.: J. Exp. Med., 165, 615 (1987)

28) Sonoda, S., Sonoda, T., Nakano, T., Kanayama, Y., Kanakura, Y., Asai, H., Yonezawa, T. and Kitamura, Y.: J. Immunol., 137, 1319 (1986)

29) Kanakura, Y., Thompson, H., Nakano, T., Yamamura, T., Asai, H., Kitamura, Y., Metcalfe, D.D. and Galli, S.J.: Blood, 72, 877 (1988) 